\title{
Activity-Dependent Acceleration of Endocytosis at a Central Synapse
}

\author{
Wei Wu, Jianhua Xu, Xin-Sheng Wu, and Ling-Gang Wu \\ National Institute of Neurological Disorders and Stroke, Bethesda, Maryland 20892
}

Accumulated evidence indicates the existence of rapid and slow endocytosis at many synapses. It has been proposed that rapid endocytosis is activated by intense stimulation when vesicle recycling needs to be speeded up to supply vesicles at hippocampal synapses. However, the evidence, as obtained with imaging techniques, which are somewhat indirect in indicating rapid endocytosis, is controversial. Furthermore, a slower time course of endocytosis is often found after more intense nerve activity, casting doubt on the role of rapid endocytosis at synapses. Here, we addressed this issue at a mammalian central synapse, the calyx of Held, using a capacitance measurement technique that provides a higher time resolution than imaging techniques. We found that rapid endocytosis with a time constant of $\sim 1-2 \mathrm{~s}$ was activated during intense nerve activity. Reducing the presynaptic calcium current or buffering the intracellular calcium with EGTA significantly inhibited rapid endocytosis, suggesting that calcium triggers rapid endocytosis. During intense stimulation, rapid endocytosis retrieved up to approximately eight vesicles per second per active zone, approximately eightfold larger than reported in the hippocampus, and thus played a dominant role during and within $3 \mathrm{~s}$ after intense stimulation. Slow endocytosis became dominant $3 \mathrm{~s}$ after intense stimulation likely because of the fall of the intracellular calcium level that deactivated rapid endocytosis. These results underscore the importance of calcium-triggered rapid endocytosis, which offers the nerve terminal the plasticity to speed up vesicle cycling during intense nerve activity.

Key words: synapse; calcium; plasticity; endocytosis; short-term facilitation; exocytosis

\section{Introduction}

The time course of vesicle endocytosis may range from $<1 \mathrm{~s}$ to $>100$ s at many synapses (Royle and Lagnado, 2003; Wu, 2004). Rapid endocytosis is apparently advantageous over slow endocytosis in recycling vesicles and sustaining synaptic transmission. This seems particularly critical for the mammalian CNS, in which only a fraction of vesicles in the nerve terminal can be mobilized for exocytosis and endocytosis in physiological stimulation conditions (Harata et al., 2001; de Lange et al., 2003). Naturally, one would expect that rapid endocytosis is turned on when fast vesicle recycling is needed to balance the rate of exocytosis during intense nerve activity. Indeed, rapid vesicle endocytosis and reuse are suggested to occur during more intense stimulation at mammalian central synapses (Klingauf et al., 1998; Sara et al., 2002). This suggestion, however, is in sharp contrast to the widely observed phenomenon that the time course of endocytosis increases in parallel with the stimulation intensity and the amount of exocytosis (for review, see $\mathrm{Wu}, 2004$ ). The reason for this apparent controversy is not clear. One of the main arguments for faster endocytosis during more intense stimulation is that vesicles

\footnotetext{
Received April 29, 2005; revised 0ct. 11, 2005; accepted Nov. 1, 2005.

This work was supported by the National Institute of Neurological Disorders and Stroke Intramural Research Program. We thank Dr. Ken Paradiso for comments on this manuscript.

Correspondence should be addressed to Ling-Gang Wu, National Institute of Neurological Disorders and Stroke, National Institutes of Health, 36 Convent Drive, Building 36, Room 1C12, Bethesda, MD 20892. E-mail: wul@ninds.nih.gov.

DOI:10.1523/JNEUROSCI.2972-05.2005

Copyright $\odot 2005$ Society for Neuroscience $\quad$ 0270-6474/05/2511676-08\$15.00/0
}

loaded with FM dyes of different rates of dissociation from the plasma membrane are destained at different time courses (Klingauf et al., 1998). However, similar destaining rates between these two dyes are found at the same synapse, the cultured hippocampal synapse, by another laboratory (Fernandez-Alfonso and Ryan, 2004). The latter result (Fernandez-Alfonso and Ryan, 2004), together with the observation of slower endocytosis after more intense stimulation at many synapses, casts the doubt on whether endocytosis and, thus, recycling is speeded up by intense stimulation to cope with the increased exocytosis at mammalian central synapses. To clear this doubt, evidence more direct than FM dye-imaging techniques is needed. Alternatively, the controversy can be reconciled by a hypothesis in which endocytosis is rapid, but with a constant rate (cannot be upregulated) and a limited capacity (approximately one vesicle per second per bouton) (Sankaranarayanan and Ryan, 2000; Fernandez-Alfonso and Ryan, 2004). According to this hypothesis, rapid endocytosis can be observed during intense stimulation, and slower time course of endocytosis after more intense stimulation is a result of saturation. As the limited capacity is saturated, this hypothesis leaves no room for plasticity of endocytosis to cope with the rate of exocytosis during stimulation.

In contrast to the prediction from the saturation hypothesis, here we reported that rapid endocytosis was turned on to cope with the rate of exocytosis during repetitive stimulation at a mammalian central nerve terminal, the calyx of Held. We used the capacitance recording technique, which provides a more direct measurement of endocytosis. We found that rapid endocy- 
tosis was activated by increased calcium influx and became the dominant mechanism to retrieve fused vesicles and maintain surface area during intense nerve activity. Rapid endocytosis gave away its dominant role to slow endocytosis after stimulation likely because of the fall of the intracellular calcium level. These results may reconcile the debate on whether intense stimulation evokes rapid or slow endocytosis.

\section{Materials and Methods}

Methods for preparing parasagittal brainstem slices from 7- to 10-d-old Wistar rats and measurements of $\mathrm{Ca}^{2+}$ currents, capacitance, and EPSCs in the medial nucleus of the trapezoid body have been described previously (Sun and Wu, 2001; Sun et al., 2002). Unless otherwise mentioned, recordings were made at room temperature $\left(22-25^{\circ} \mathrm{C}\right)$ in a bath solution that pharmacologically isolated $\mathrm{Ca}^{2+}$ currents. This solution contained the following (in mM): $105 \mathrm{NaCl}, 20$ tetraethylammonium-Cl, $2.5 \mathrm{KCl}, 1$ $\mathrm{MgCl}_{2}, 2 \mathrm{CaCl}_{2}, 25 \mathrm{NaHCO}_{3}, 1.25 \mathrm{NaH}_{2} \mathrm{PO}_{4}, 25$ dextrose, 0.4 ascorbic acid, 3 myo-inositol, 2 sodium pyruvate, 0.001 tetrodotoxin, $0.13,4$ diaminopyridine, $\mathrm{pH} 7.4$, when bubbled with $95 \% \mathrm{O}_{2}$ and $5 \% \mathrm{CO}_{2}$. If not mentioned, the presynaptic pipette $(2.5-4.5 \mathrm{M} \Omega$ ) solution contained the following (in mM): 125 Cs-gluconate, $20 \mathrm{CsCl}, 4 \mathrm{MgATP}, 10 \mathrm{Na}_{2}-$ phosphocreatine, 0.3 GTP, 10 HEPES, 0.05 BAPTA, pH 7.2, adjusted with $\mathrm{CsOH}$. When BAPTA was replaced by $10 \mathrm{~mm}$ EGTA, Cs-gluconate concentration was reduced to maintain the osmolarity $(\sim 305-315$ mOsm). Postsynaptic pipette ( $2-3 \mathrm{M} \Omega$ ) solution was similar to the presynaptic standard pipette solution with $\mathrm{Cs}^{+}$replaced by $\mathrm{K}^{+}$and BAPTA replaced by $0.5 \mathrm{~mm}$ EGTA. The holding potential for both presynaptic and postsynaptic recordings was $-80 \mathrm{mV}$. Data were expressed as mean \pm SEM.

For capacitance measurements (EPC-9 amplifier, PULSE program; HEKA, Lambrecht, Germany), the sinusoidal stimulus was $1000 \mathrm{~Hz}$ with a peak-to-peak voltage of $60 \mathrm{mV}$. We previously selected calyces showing a single exponential decay of their passive current transients (Sun et al., 2002). In this study, we did not perform any preselection, because similar results were obtained from capacitance measurements in calyces showing a monoexponential or biexponential passive current transient (Taschenberger et al., 2002; Sun et al., 2004). In the present work, the intensity of stimulation was generally higher than that previously used to compare capacitance changes (Taschenberger et al., 2002; Sun et al., 2004). The most intense stimulation we used was $10-20$ pulses of $20 \mathrm{~ms}$ depolarization from -80 to $+10 \mathrm{mV}$ at $1-10 \mathrm{~Hz}$. With these intense stimuli, similar results were obtained from calyces showing a monoexponential or biexponential passive current transient (for detail, see supplemental material and Fig. S1, available at www.jneurosci.org). Thus, throughout this study, we did not differentiate results obtained from calyces showing a monoexponential or biexponential passive current transient.

Slow baseline capacitance drift was sometimes observed, which may affect measurements of slow but not rapid endocytosis. To avoid such influence, we discarded the data if the baseline drift measured within 10 to $60 \mathrm{~s}$ before stimulation was $\geq 15 \mathrm{fF} / \mathrm{s}$ or predicted a shift of $>30 \%$ of the capacitance jump in $40 \mathrm{~s}$. For data used in this study, more than two-thirds did not require baseline correction, others were fit with a linear regression line in the capacitance baseline, which were subtracted from the data.

To avoid contamination of capacitance artifacts, we measured the amount of exocytosis or endocytosis at $250 \mathrm{~ms}$ after a $20 \mathrm{~ms}$ depolarization, a train of 20 ms depolarization, and a train of AP-e. For single AP-e and $1 \mathrm{~ms}$ depolarization, we neglected the first $200 \mathrm{~ms}$ after stimulation (Fig. S3 B, C, available at www.jneurosci.org as supplemental material). For measurements of the amplitude of capacitance artifacts in the presence of BoNT/C, measurements started at $10 \mathrm{~ms}$ after the stimulus (see Fig. 2E) (Fig. S3A, available at www.jneurosci.org as supplemental material).

\section{Results}

Acceleration of endocytosis

A $10-20 \mathrm{~ms}$ pulse from -80 to $+10 \mathrm{mV}$ or a high-frequency train of action potential-equivalent stimuli (AP-e) ( $1 \mathrm{~ms}$ from -80 to
$7 \mathrm{mV}$ ) depleted the readily releasable pool at calyces of Held ( $\mathrm{Wu}$ and Borst, 1999; Sun and Wu, 2001). For convenience, we used a depolarizing pulse ( $20 \mathrm{~ms}$ from -80 to $+10 \mathrm{mV}$, unless otherwise indicated) instead of an AP-e train to deplete the readily releasable pool. This pulse increased the capacitance by $473 \pm 68$ $\mathrm{fF}(n=9)$. The jump decayed monoexponentially with a mean time constant $(\tau)$ of $12.0 \pm 3.1 \mathrm{~s}(n=9)$ (Fig. $1 A)$, which reflects endocytosis (Sun and $\mathrm{Wu}, 2001$ ). The time constant of endocytosis varied significantly among different calyces. For nine calyces recorded, the time constant ranged from 4.5 to $25 \mathrm{~s}$. Wide variation seems a common feature of endocytosis, because the time course of endocytosis also varied significantly after an AP-e (Fig. $\mathrm{S} 3 B$, available at www.jneurosci.org as supplemental material).

When the $20 \mathrm{~ms}$ pulse was repeated 10 times at $10 \mathrm{~Hz}$, which was much stronger than most stimuli used in our previous study (Sun et at., 2002), the capacitance jumped by $1287 \pm 102 \mathrm{fF}$ ( $n=$ 11) (Fig. $1 \mathrm{~B}$ ). The decay after the jump was fit well, as judged by eye, with a biexponential function, with time constants of $1.4 \pm$ $0.4 \mathrm{~s}(n=11$; amplitude, $407 \pm 48 \mathrm{fF})$ and $18.4 \pm 1.7 \mathrm{~s}(n=11$; amplitude, $880 \pm 58 \mathrm{fF}$ ), respectively (Fig. $1 B$ ). Both the rapid (Fig. $1 B$, right) and the slow (Fig. $1 B$, left) component of the capacitance decay were not paralleled with, and thus not caused by, changes in the membrane conductance or the series conductance (Fig. 2C) (Fig. S1 B, available at www.jneurosci.org as supplemental material). These results suggest that repetitive depolarization induced a kinetically rapid form of endocytosis. Such an activity-dependent acceleration of endocytosis could be repeated every 1-2 min for up to $15 \mathrm{~min}$ of whole-cell recordings. (Fig. S2, available at www.jneurosci.org as supplemental material).

To determine how rapid endocytosis developed during stimulation, we decreased the frequency from 10 to $1 \mathrm{~Hz}$. The capacitance decay between each pulse was not paralleled with and thus not caused by changes in the membrane conductance or the series conductance (Figs. $1 C$, right, $2 D$ ) (Fig. S1 D, available at www. jneurosci.org as supplemental material). The rate of endocytosis between each pulse was measured as the amplitude of the capacitance decay during $250-1000 \mathrm{~ms}$ after a pulse divided by $750 \mathrm{~ms}$. We did not measure the first $250 \mathrm{~ms}$ of capacitance values, because it was contaminated by artifacts (reasons were presented later in Fig. $2 \mathrm{E}$ ). During the $1 \mathrm{~Hz}$ train, the rate of endocytosis increased from $28 \pm 17 \mathrm{fF} / \mathrm{s}$ after the first pulse to a plateau level of $208 \pm 38 \mathrm{fF} / \mathrm{s}(n=7)$ after the sixth pulse (Fig. 1C,D, top), suggesting that repeated stimulation speeds up endocytosis. After 10 pulses at $1 \mathrm{~Hz}$, the capacitance increased by $1607 \pm 136 \mathrm{fF}(n=$ 7 ) and decayed biexponentially with time constants of $1.1 \pm 0.2 \mathrm{~s}$ (amplitude, $217 \pm 33 \mathrm{fF}$ ) and $23.1 \pm 2.2 \mathrm{~s}(n=7$; amplitude, $1390 \pm 88 \mathrm{fF}$ ) (Fig. 1C), respectively. As calculated from the fitted exponential function in each recorded calyx, the contributions of the rapid and the slow component of endocytosis in the first second after the train were $129 \pm 17$ and $72 \pm 11 \mathrm{fF} / \mathrm{s}(n=7)$, respectively. The sum of these two numbers matched closely the plateau level $(208 \pm 38 \mathrm{fF} / \mathrm{s} ; n=7)$ of the endocytosis rate during the $1 \mathrm{~Hz}$ train. The larger contribution by the rapid $(129 \pm 17$ $\mathrm{fF} / \mathrm{s})$ versus the slow $(72 \pm 11 \mathrm{fF} / \mathrm{s})$ component of endocytosis suggests a dominant role of rapid endocytosis during stimulation.

Acceleration of endocytosis (Fig. 1D, top) increased the number of endocytosed vesicles (Fig. $1 D$, triangles), which paralleled the increased number of exocytosed vesicles (Fig. $1 D$, squares) and thus kept the net membrane capacitance (Fig. $1 D$, circles) from further increase during stimulation (see figures for calculation methods). At the end of the $1 \mathrm{~Hz}$ train, the accumulated exocytosis was $5.7 \pm 0.4 \mathrm{pF}(n=7)$ (Fig. $1 D$, squares), consider- 
ing that a vesicle capacitance is $\sim 0.065 \mathrm{fF}$ (Sun et al., 2002), $5.7 \mathrm{pF}$ corresponds to $\sim 88,000$ vesicles, which is in the same order as the total number $(\sim 77,000-188,000)$ of vesicles in a calyx (Sätzler et al., 2002; de Lange et al., 2003). However, the net capacitance increase was only $2.1 \pm 0.1 \mathrm{pF}(n=7)$ (Fig. $1 D$, circles). Thus, $3.6 \pm 0.6 \mathrm{pF}(n=7)$ (Fig. 1D, triangles) of fused vesicles or $\sim 63 \%$ of exocytosed vesicles were retrieved during the train, indicating a critical role of rapid endocytosis during intense nerve activity.

\section{Rapid capacitance decay} reflects endocytosis

In the presence of a botulinum neurotoxin that blocks exocytosis, $10 \mathrm{~ms}$ depolarization induces a transient capacitance artifact ( $\sim 64 \mathrm{fF}$, with $\tau=\sim 227 \mathrm{~ms}$ ) at calyces (Yamashita et al., 2005). Here, we used two approaches to determine whether the rapid capacitance decay during stimulation is an artifact. First, we compared the capacitance jump and the AMPA receptor-mediated EPSC evoked at the same synapse. Kynurenate (1 $\mathrm{mm}$ ) was added to the bath solution to relieve AMPA receptor saturation and desensitization at calyx-type synapses (Wong et al., 2003). The fractional changes in the EPSC charge (integral for the first $100 \mathrm{~ms}$ after depolarization) and the capacitance jump were paralleled and similar during $20 \mathrm{de}$ polarizing pulses at $1 \mathrm{~Hz}$ (Fig. $2 A, B$ ). At the end of the train, the EPSC charge and the capacitance jump decreased to $27 \pm$ $8 \%$ and $31 \pm 7 \%(n=4)$ (Fig. $2 A, B)$ of the first response, respectively (note that different depression of the capacitance jump observed in Figs. $1 C$ and $2 A$ were attributable to different calyces). The slightly higher value of the capacitance jump than the EPSC charge (Fig. 2B) was perhaps a result of incomplete relief of receptor saturation and desensitization. Kynurenate has been shown to effectively block desensitization and receptor saturation during a train of action potentials at $200 \mathrm{~Hz}$ for $1 \mathrm{~s}$ (Wong et al., 2003), which depletes the readily releasable pool (Wong et al., 2003). The stimulation used in this work was apparently more intensive. We used a train of $20 \mathrm{~ms}$ pulses at $1 \mathrm{~Hz}$. Each $20 \mathrm{~ms}$ pulse was sufficient to deplete the readily releasable pool (Sun and $\mathrm{Wu}, 2001)$. Thus, the incomplete relief of receptor saturation and desensitization could not be ruled out. Nevertheless, the approximately similar capacitance jump and the EPSC charge during the $1 \mathrm{~Hz}$ train (Fig. $2 \mathrm{~B}$ ) suggest that the capacitance jump mostly reflected exocytosis and thus the decay during the train indicated endocytosis.

The second approach was to add $1 \mu \mathrm{m}$ botulinum neurotoxin C (BoNT/C; Wako Chemicals, Richmond, VA) into the pipette solution to block exocytosis (Xu et al., 1998). The bath was heated to $33-34^{\circ} \mathrm{C}$ to facilitate the block (Xu et al., 1998). At 1-3 min after break-in, the capacitance jump induced by 10 depolarizing pulses at $10 \mathrm{~Hz}$ was $1391 \pm 169 \mathrm{fF}(n=6)$, which decayed with a
B

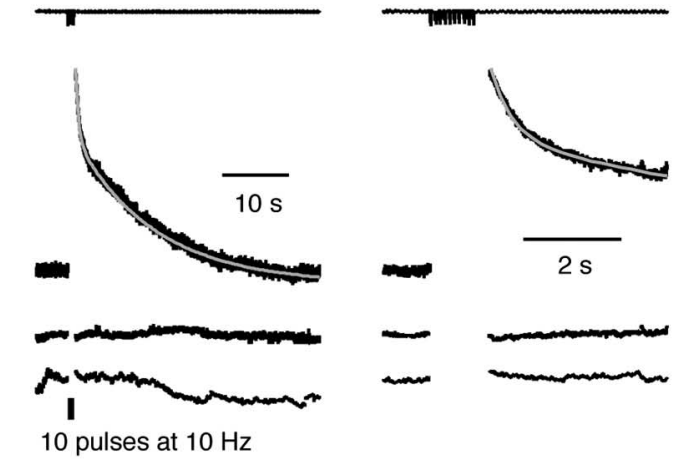

D

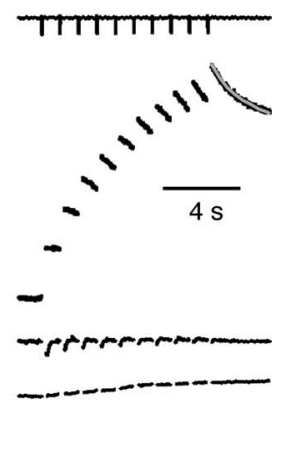

Figure 1. Rapid capacitance decay induced by repetitive $20 \mathrm{~ms}$ depolarization. $\boldsymbol{A}$-C, Sampled changes in calcium current (ICa), membrane capacitance $(\mathrm{Cm})$, membrane conductance $(\mathrm{Gm})$, and series conductance $(\mathrm{Gs})$ induced by three different stimuli (arows or bars): one depolarizing pulse (20 ms from -80 to $+10 \mathrm{mV} ; \boldsymbol{A}), 10$ pulses at $10 \mathrm{~Hz}(\boldsymbol{B})$, or $1 \mathrm{~Hz}(\boldsymbol{C})$. The capacitance decay

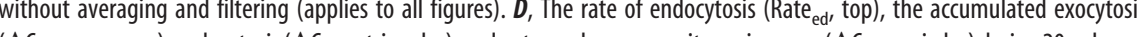
$\left(\Delta \mathrm{Cm}_{\mathrm{exo}^{\prime}}\right.$ squares), endocytosis ( $\Delta \mathrm{Cm}_{\mathrm{ed}}$, triangles), and net membrane capacitance increase $\left(\Delta \mathrm{Cm}_{\mathrm{net}}\right.$, circles $)$ during 20 pulses of

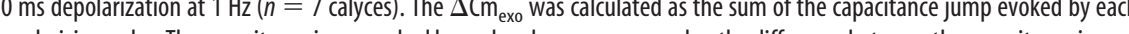
diately before and $250 \mathrm{~ms}$ after depolarization, plus the amount of capacitance that decayed within the first $250 \mathrm{~ms}$ after depolarization, which was estimated as the linear extrapolation of the capacitance decay between 250 and 1000 ms after depolarization. The $\Delta \mathrm{Cm}_{\text {net }}$ was measured as the capacitance change at $1 \mathrm{~s}$ after each pulse from the baseline before the train. The difference between $\Delta \mathrm{Cm}_{\mathrm{exo}}$ and $\Delta \mathrm{Cm}_{\text {net }}$ was $\Delta \mathrm{Cm}_{\mathrm{ed}}$. These calculations apply to Figure 3 .

biexponential function with time constants of $1.3 \pm 0.3 \mathrm{~s}(n=6$; amplitude, $799 \pm 149 \mathrm{fF})$ and $8.8 \pm 2.4 \mathrm{~s}(n=6)$, respectively (Fig. $2 C$, black). The rapid component was $57 \pm 8 \%(n=6)$ of the total capacitance jump, which was significantly larger than that $(32 \pm 5 \% ; n=11)$ at room temperature. The $\tau$ of the slow $(8.8 \pm 2.4 \mathrm{~s} ; n=6)$ but not the rapid component was significantly smaller than that $(18.4 \pm 1.7 \mathrm{~s} ; n=11 ; p<0.01)$ in control. Thus, the high temperature speeded up endocytosis, consistent with results obtained in hippocampal synapses (Fernandez-Alfonso and Ryan, 2004). Similar results were obtained with a control pipette solution at $33-34^{\circ} \mathrm{C}(n=5$; data not shown $)$.

At $10-15 \mathrm{~min}$ after break-in with a pipette containing BoNT/C (Fig. 2C, gray), the capacitance jump induced by 10 depolarizing pulses at $10 \mathrm{~Hz}$ was reduced to $116 \pm 15 \mathrm{fF}(n=5)$ or $9 \pm 2 \%$ of that at $1-3$ min after break-in. The amplitude of the rapid component of the capacitance decay was reduced to $1 \pm 1 \%$ ( $n=5$; in four calyces, no rapid component was evident) (Fig. $2 C)$ of that at 1-3 min after break-in. These decreases were not attributable to run down (Fig. S2, available at www.jneurosci.org as supplemental material). Similarly, the rapid capacitance decay 
A

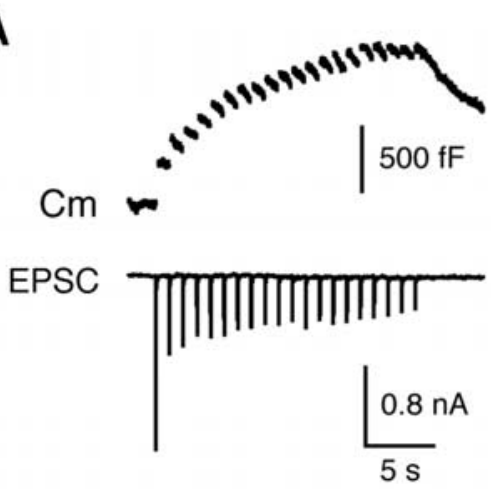

B

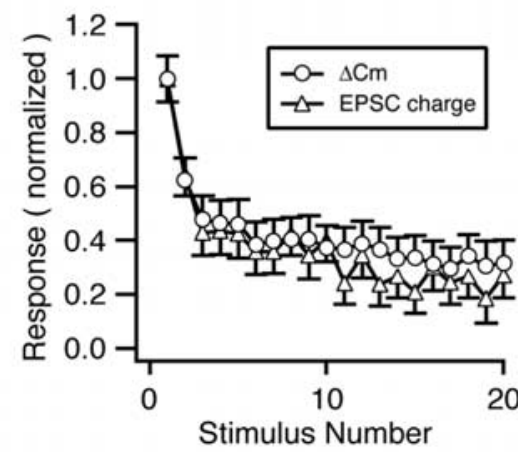

C
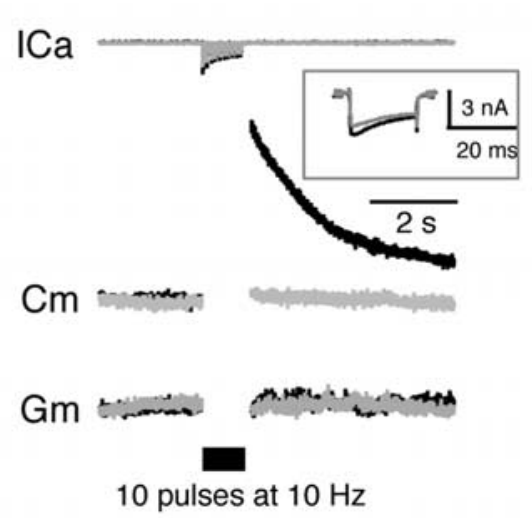

$\mathbf{E}$
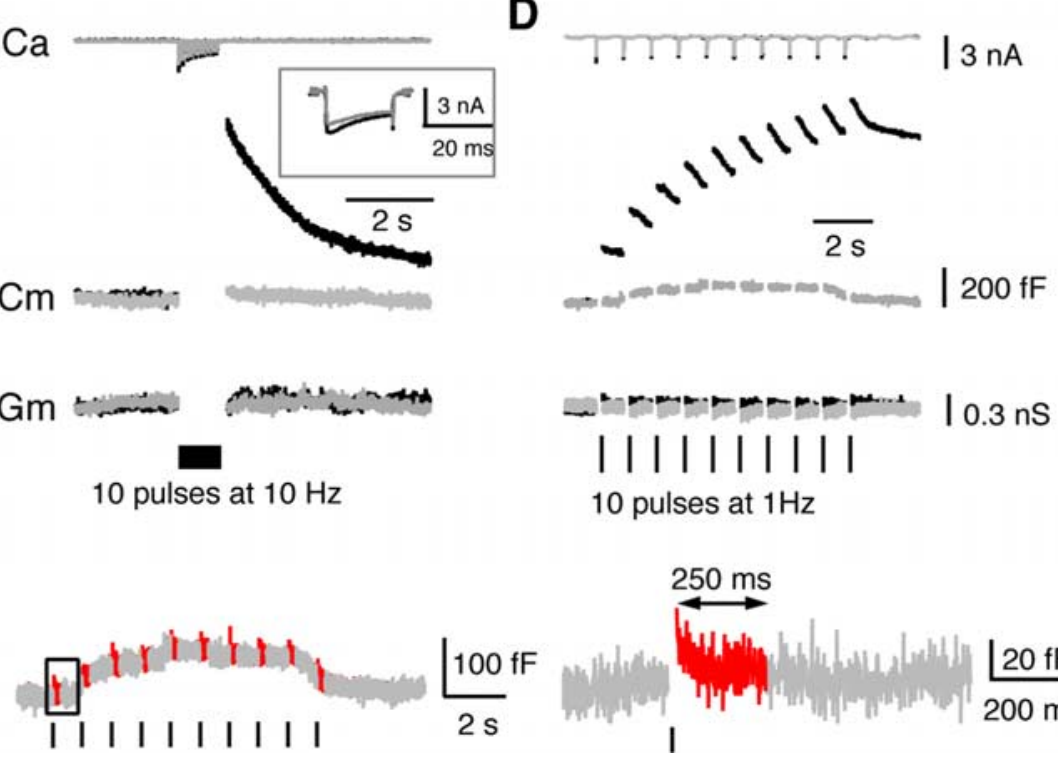

Figure 2. Rapid capacitance decay reflects endocytosis. $A$, Sampled (m and EPSC induced by 20 depolarizing pulses at $1 \mathrm{~Hz}$ with a control pipette solution at room temperature. The bath solution included $1 \mathrm{~mm}$ kynurenate. $\boldsymbol{B}$, The capacitance jump (circles) and the EPSC charge (triangles) caused by each depolarizing stimulus during a train of $20 \mathrm{~ms}$ depolarization at $1 \mathrm{~Hz}(n=6)$ in control conditions. Data were obtained from six synapses, including the one shown in $A$. Data were normalized to the first response during the train. $\boldsymbol{C}$, $\boldsymbol{D}$, Sampled ICa, $\mathrm{Cm}$, and $\mathrm{Gm}$ induced by 10 pulses of 20 ms depolarization at $10 \mathrm{~Hz}(\boldsymbol{C})$ or $1 \mathrm{~Hz}$ (D) at 2-3 min (black) and $12-14 \mathrm{~min}$ (gray) after break-in with a pipette containing $1 \mu \mathrm{m} \mathrm{BoNT} / \mathrm{C}$ at 33-34 $4^{\circ} \mathrm{C}$. The inset in C shows ICa induced by the first pulse in larger scales. $\boldsymbol{E}$, The capacitance trace in the presence of BoNT/C in $\boldsymbol{D}$ (gray) is shown without deleting the first $250 \mathrm{~ms}$ of data (red) after each pulse during the train (left). The capacitance response to the first pulse (square in left) is shown in the right in larger scale. The trace in red indicates the first $250 \mathrm{~ms}$, whereas the trace in gray indicates the time between 250 and $1000 \mathrm{~ms}$ after a depolarizing pulse.

during 10 depolarizing pulses at $1 \mathrm{~Hz}$, as measured between the ninth and the 10th pulses, was $277 \pm 41 \mathrm{fF} / \mathrm{s}(n=4)$ (Fig. $2 D$, black) at 1-3 min after break-in but reduced to $34 \pm 6 \mathrm{fF} / \mathrm{s}(n=$ 4) (Fig. $2 D$, gray) at $10-15 \mathrm{~min}$ after break-in with a pipette containing BoNT/C. We concluded that capacitance jumps were caused by exocytosis, and thus the rapid capacitance decay reflected endocytosis.

To avoid capacitance transient artifacts (Yamashita et al., 2005), we neglected the first $250 \mathrm{~ms}$ of the capacitance change evoked by a $20 \mathrm{~ms}$ depolarization. The reason is given below. At 10-15 min after break-in with a pipette containing BoNT/C, a single depolarizing pulse $(20 \mathrm{~ms})$ induced negligible capacitance jump $(12 \pm 5 \mathrm{fF} ; n=4)$ as measured $250 \mathrm{~ms}$ after stimulation (Fig. 2D, gray). However, as measured $10 \mathrm{~ms}$ after stimulation, we observed a transient capacitance change (50 $\pm 15 \mathrm{fF} ; n=4)$ with a time constant of $233 \pm 76 \mathrm{~ms}(n=4)$ after a $20 \mathrm{~ms}$ depolarization (Fig. $2 E$ ). This result was consistent with the capacitance artifact $(\sim 64 \mathrm{fF}$, with $\tau=\sim 227 \mathrm{~ms}$ ) previously ob- served after a $10 \mathrm{~ms}$ depolarization in the presence of BoNT/E, which also abolishes exocytosis (Yamashita et al., 2005). Thus, except results in Figure $2 E$, we did not measure and show the capacitance for the first $250 \mathrm{~ms}$ after a $20 \mathrm{~ms}$ depolarization or a train of depolarizing pulses throughout the present study.

The observation of a transient capacitance $\operatorname{artifact}(\tau=\sim 227-233 \mathrm{~ms}$ ) (Yamashita et al., 2005) implies that our previous report (Sun et al., 2002) on fast endocytosis with a $\tau$ of $\sim 100-300 \mathrm{~ms}$ after mild stimulation, such as an AP-e, may be contaminated with artifacts. We reexamined this issue and found that indeed the time constant was underestimated. Our current estimate was $2.2 \pm 0.6 \mathrm{~s}$ after an AP-e $(n=8)$ (Fig. S3B, available at www. jneurosci.org as supplemental material). Because the current study does not focus on endocytosis induced by mild stimulation, these results were described in detail in the supplemental material (available at www.jneurosci.org).

\section{Calcium triggers rapid endocytosis}

Calcium has been suggested to facilitate, inhibit, or not affect endocytosis (for review, see $\mathrm{Wu}, 2004)$. To determine whether calcium mediates facilitation of endocytosis, we manipulated calcium by two approaches. First, we lowered the depolarization voltage from a control of 90 $\mathrm{mV}$ (from -80 to $+10 \mathrm{mV}$ ) to $75 \mathrm{mV}$ (from -80 to $-5 \mathrm{mV}$ ). The calcium current amplitude during the first $20 \mathrm{~ms}$ depolarizing pulse was decreased from $2.66 \pm 0.13 \mathrm{nA}(n=9)$ (Fig. $1 A$, inset) to $0.69 \pm 0.10 \mathrm{nA}(n=6)$ (Fig. $3 A$, inset). The endocytosis rate between neighboring pulses reached a plateau level of $52 \pm 6 \mathrm{fF} / \mathrm{s}$ $(n=5)$ at the fifth depolarization during the $1 \mathrm{~Hz}$ train (Fig. 3B, black diamonds), which was approximately fourfold less than that $(208 \pm 38 \mathrm{fF} / \mathrm{s} ; n=7)$ in control (Fig. 3B, gray diamonds). After the train, the capacitance jump (805 $\pm 156 \mathrm{fF})$ decayed mostly monoexponentially with a time constant of $19.0 \pm 1.6 \mathrm{~s}(n=5)$ (Fig. $3 A)$, which was similar to the slower component of endocytosis in control. This slow endocytosis would generate a capacitance decay of $42 \pm 6 \mathrm{fF}(n=5)$ in the first second after the train, which was in the same range as the endocytosis rate ( $52 \pm 6 \mathrm{fF} / \mathrm{s} ; n=5)$ during stimulation. Thus, reducing calcium currents primarily blocked rapid, but not slow, endocytosis during stimulation.

Similar results were obtained when 10 mm EGTA was added to the pipette solution (Fig. 3C,D), which effectively buffered residual calcium increase induced by a $20 \mathrm{~ms}$ depolarization (Wu and Borst, 1999). For example, the maximal rate of endocytosis during 10-20 depolarizing pulses at $1 \mathrm{~Hz}$ was $69 \mathrm{fF} / \mathrm{s}$ (Fig. 3C; summarized in Fig. 3D, black diamonds), which was much less than that in control (Fig. 3D, gray diamonds). After the train, the capacitance jump $(850 \pm 85 \mathrm{fF})$ induced by 10 pulses at $1 \mathrm{~Hz}$ 
decayed primarily monoexponentially with a time constant of $14.3 \pm 1.5 \mathrm{~s}(n=4)$ (Fig. 3C), which was not larger than that of the slower component of endocytosis in control (23.1 $\pm 2.2 \mathrm{~s}$; amplitude, $1390 \pm$ $88 \mathrm{fF} ; n=7)$. Together, our results suggest that the increased intracellular calcium triggers rapid endocytosis during and after stimulation. This suggestion predicts that higher frequencies of stimulation induce larger amplitudes of rapid endocytosis by increasing the intracellular calcium concentration to a higher level. Indeed, the amplitude of rapid endocytosis after $10 \mathrm{de}$ polarizing pulses at $10 \mathrm{~Hz}(407 \pm 48 \mathrm{fF}$; $n=11$ ) (Fig. $1 B$ ) was higher than that at 1 $\mathrm{Hz}(217 \pm 33 \mathrm{fF} ; n=7)$ (Fig. 1C). In addition, EGTA did not slow down the slow component of endocytosis after stimulation, which is consistent with our previous report that $10 \mathrm{~mm}$ EGTA did not significantly affect endocytosis with a time constant of $\sim 10$ s (Sun et al., 2002).

Reducing calcium currents or buffering calcium influx with EGTA reduced the net capacitance increase (Fig. $3 B, D$ ). Could a reduction in the net capacitance increase block rapid endocytosis? This possibility is highly unlikely for two reasons. First, the endocytosis rate reached a plateau level after approximately the fifth pulse (Fig. $3 B$, top), whereas the net capacitance increase continued to climb up (Fig. $3 B$, circles; example in Fig. $4 A$ ). Second, we plotted the endocytosis rate versus the net capacitance increase during pulse trains at $1 \mathrm{~Hz}$ in control (Fig. $4 B$, circles) (90 $\mathrm{mV}$ depolarization) and in the reduced calcium current condition (Fig. $4 B$, triangles) (75 mV depolarization). When the net capacitance increase was $>1 \mathrm{pF}$, for the same net capacitance increase, the endocytosis rate was much faster in control than in the reduced calcium condition (Fig. 4B). Similarly, with the same net capacitance increase $>1 \mathrm{pF}$, the endocytosis rate was much faster in control (Fig. $4 C$, circles) than in the presence of $10 \mathrm{~mm}$ EGTA in the pipette (Fig. 4C, triangles).

\section{Endocytosis is accelerated during repetitive AP-e}

In the experiments shown thus far, intense stimulation was mimicked by repeated $20 \mathrm{~ms}$ depolarizing pulses because of the convenience in controlling calcium currents and release. In the following, we showed that the same principles (Figs. 1,3) applied to high-frequency trains of AP-e ( $1 \mathrm{~ms}$ from -80 to $+7 \mathrm{mV})$. Applying $50 \mathrm{AP}-\mathrm{e}$ at $100 \mathrm{~Hz}$ increased the capacitance by $710 \pm 56$ $\mathrm{fF}$, which decayed largely monoexponentially with a time constant of $16.4 \pm 2.1 \mathrm{~s}(n=7)$ (Fig. 5A). Applying 200 AP-e at 100 $\mathrm{Hz}$, however, increased the capacitance by $1362 \pm 122 \mathrm{fF}$, which decayed biexponentially with time constants of $1.9 \pm 0.4 \mathrm{~s}$ (337 \pm $27 \mathrm{fF}$ ) and $19.6 \pm 3.9 \mathrm{~s}(n=6)$ (Fig. $5 B)$, respectively. These results suggest that longer $\mathrm{AP}$-e trains activate rapid endocytosis.

When a burst of $50 \mathrm{AP}-\mathrm{e}$ at $100 \mathrm{~Hz}$ was repeated five times at $1 \mathrm{~Hz}$, the endocytosis rate between neighboring bursts increased from $64 \pm 8$ to $219 \pm 18 \mathrm{fF} / \mathrm{s}(n=8)$ after the fifth burst (Fig. $5 C$, right). After the train, the capacitance jump $(1344 \pm 155 \mathrm{fF})$ decayed biexponentially with time constants of $1.4 \pm 0.3 \mathrm{~s}(284 \pm$ $44 \mathrm{fF})$ and $18.8 \pm 2.6 \mathrm{~s}(n=8)$, respectively (Fig. $5 C$, left). Similarly, when a burst of $50 \mathrm{AP}-\mathrm{e}$ at $30 \mathrm{~Hz}$ was repeated five times at
B

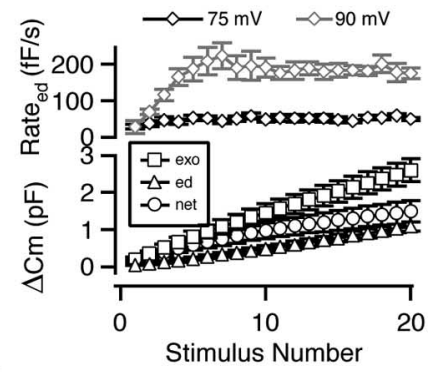

D

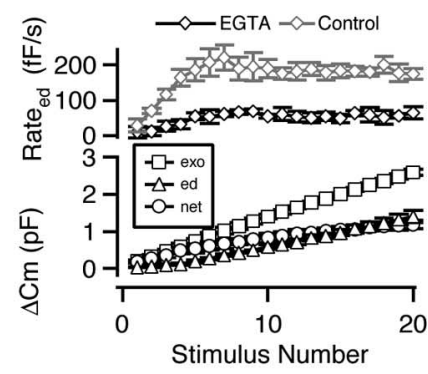

Figure 3. Calcium triggers rapid endocytosis. $\boldsymbol{A}$, Left, Sampled ICa and ( $\mathrm{m}$ induced by 10 pulses of $20 \mathrm{~ms}$ depolarization to -5 $\mathrm{mV}$ at $1 \mathrm{~Hz}$. The capacitance decay after the train was fit with a monoexponential function (gray; $\tau=17.9 \mathrm{~s}$ ). The inset shows ICa induced by the first pulse in larger scales. Right, Same traces as in the left, but in a larger time scale. $\boldsymbol{B}$, The rate of endocytosis (top

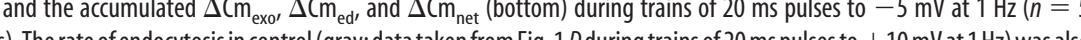
was 16.4

$0.4 \mathrm{~Hz}$, the endocytosis rate between neighboring bursts increased from $28 \pm 6$ to $116 \pm 21 \mathrm{fF} / \mathrm{s}(n=8)$ after the fifth burst (Fig. $5 D$, right). After the train, the capacitance jump (735 \pm 126 $\mathrm{fF})$ decayed biexponentially with time constants of $1.3 \pm 0.3 \mathrm{~s}$ $(136 \pm 35 \mathrm{fF})$ and $18.1 \pm 2.2 \mathrm{~s}(n=8)$, respectively (Fig. 5D, left). The amplitude of the rapid component after the $30 \mathrm{~Hz}$ train $(136 \pm 35 \mathrm{fF} ; n=8)$ (Fig. 5D) was smaller than that after the 100 $\mathrm{Hz}$ train (284 $\pm 44 \mathrm{fF} ; n=8)$ (Fig. $5 C$ ) likely for two reasons. First, $50 \mathrm{AP}$-e at $30 \mathrm{~Hz}$ lasted for $1.63 \mathrm{~s}$, during which a significant portion of rapid endocytosis could take place but could not be detected. Second, the intracellular calcium level during a $30 \mathrm{~Hz}$ train might not be as high as during $100 \mathrm{~Hz}$ train. Finally, addition of $10 \mathrm{~mm}$ EGTA into the pipette solution blocked the rapid component of endocytosis after $200 \mathrm{AP}$-e at $100 \mathrm{~Hz}$ (Fig. $5 E)(n=$ 6), similar to that after repetitive $20 \mathrm{~ms}$ depolarization (Fig. 3).

\section{The relationship between the time constant and the} amplitude of endocytosis

In the present work, we have shown the time constants and the amplitudes of rapid and/or slow components of endocytosis in a variety of stimulation conditions. These results are summarized in Table 1. The endocytic time constant is slower after more intensive stimulation and is approximately linearly proportional to the amount of exocytosis at nerve terminals ( $\mathrm{Wu}$ and Betz, 1996; Sankaranarayanan and Ryan, 2000). At calyces, this relationship was observed when the stimulation intensity was limited to $<20$ ms depolarization (Sun et al., 2002; Yamashita et al., 2005). When the stimulation intensity was increased beyond 20 ms depolarization, we showed here that a rapid component of endocytosis was activated (Fig. 1). However, if the time constant of endocytosis (when there was only a single component of endocytosis) and the time constant of the slower component of endocytosis (when there were two components of endocytosis) 


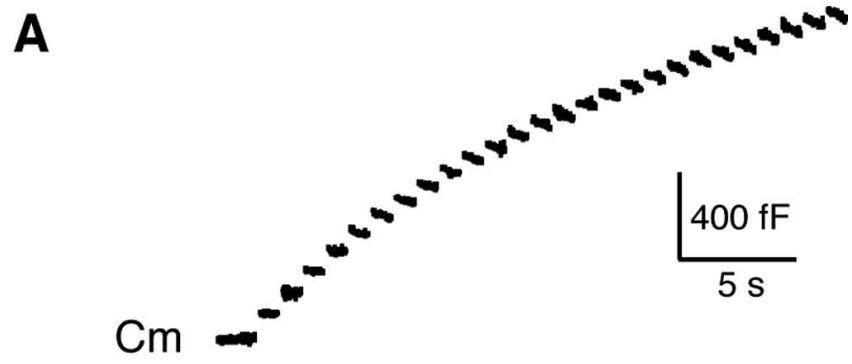

B
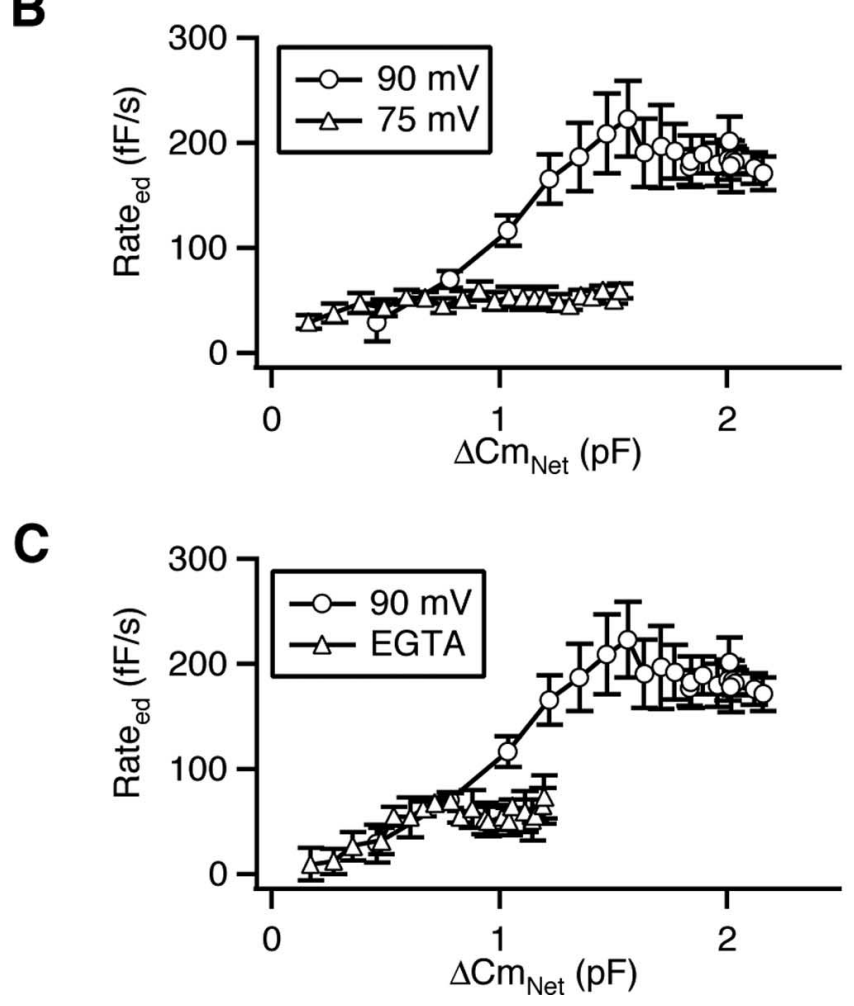

Figure 4. Acceleration of endocytosis during stimulation is not caused by the net capacitance increase. $A$, Sampled Cm induced by 20 pulses of $20 \mathrm{~ms}$ depolarization to $-5 \mathrm{mV}$ at $1 \mathrm{~Hz}$. Capacitance decay between depolarizing pulses (gaps) did not speed up significantly after the 10th depolarizing pulse, whereas the net capacitance value continued to increase. $\boldsymbol{B}$, The rate of endocytosis ( Rate $_{\text {ed }}$ ) is plotted against $\Delta \mathrm{Cm}_{\text {net }}$ during trains of $20 \mathrm{~ms}$ depolarization by $75 \mathrm{mV}$ (from -80 to $-5 \mathrm{mV}$; triangles; $n=5$; data from Fig. $3 B$ ) or by $90 \mathrm{mV}$ (from -80 to $+10 \mathrm{mV}$; circles; $n=7$; data from Fig. 1D) at $1 \mathrm{~Hz}$. C, The rate of endocytosis (Rate ed $_{\text {) }}$ is plotted against $\Delta \mathrm{Cm}_{\text {net }}$ during trains of $20 \mathrm{~ms}$ depolarization by $90 \mathrm{mV}$ at $1 \mathrm{~Hz}$ in the presence of $10 \mathrm{~mm}$ EGTA in the pipette solution (triangles; $n=4$; data from Fig. 3D) or in control pipette solution (circles; $n=7$; data from Fig. 1D).

were plotted versus their corresponding capacitance amplitude after a variety of stimuli, the approximately linear relationship still held (Fig. 6). These time constants and stimuli included the slower component of endocytosis after 10 depolarizing pulses at 1 and $10 \mathrm{~Hz}$ (Fig. $1 B, C$ ), endocytosis after a $20 \mathrm{~ms}$ depolarization (Fig. $1 A$ ), and after a $1 \mathrm{~ms}$ depolarization to +7 or $15-30 \mathrm{mV}$ (Fig. S3, available at www.jneurosci.org as supplemental material), endocytosis after $50 \mathrm{AP}$-e at $100 \mathrm{~Hz}$ (Fig. 5A), and the slower component of endocytosis after $200 \mathrm{AP}$-e at $100 \mathrm{~Hz}$ (Fig. 5B), 50 $\mathrm{AP}-\mathrm{e}$ at $100 \mathrm{~Hz}$ repeated five times at $1 \mathrm{~Hz}$ (Fig. $5 \mathrm{C}$ ), and $50 \mathrm{AP}-\mathrm{e}$ at $30 \mathrm{~Hz}$ repeated five times at $0.4 \mathrm{~Hz}$ (Fig. $5 D$ ). The potential meaning of this linear relationship (Fig. 6) was discussed in the Discussion section.
A

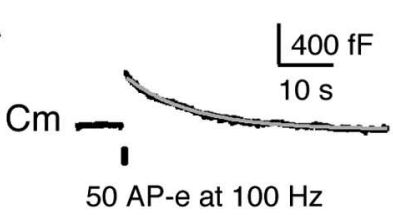

B

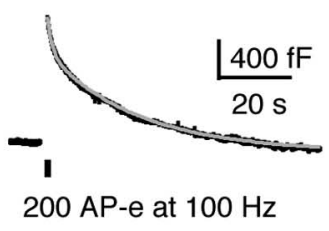

C
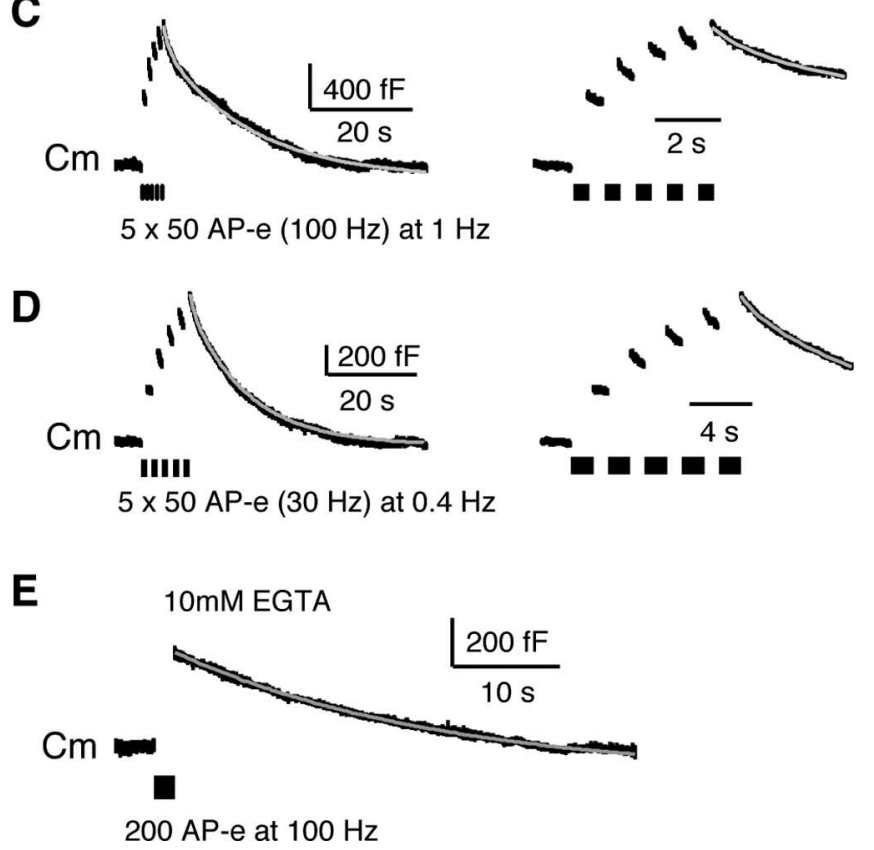

Figure 5. Rapid endocytosis induced by AP-e trains. $A, B$, Sampled $C m$ induced by $50(A)$ or 200 (B) AP-e at $100 \mathrm{~Hz}$ (bars). The capacitance decay was fit with a monoexponential function (gray; $\tau=13.1 \mathrm{~s}$ ) after $50 \mathrm{AP}$-e $(\boldsymbol{A})$ and a biexponential function [gray; $\tau_{1}=1.8 \mathrm{~s}$ (377 fF), $\tau_{2}=24.0 \mathrm{~s}$ (1020 fF)] after $200 \mathrm{AP}$-e (B). Data were from the same synapse. C, Left, Sampled $\mathrm{Cm}$ induced by five bursts at $1 \mathrm{~Hz}$. Each burst consisted of $50 \mathrm{AP}$-e at $100 \mathrm{~Hz}$. The capacitance decay was fit with a biexponential function [gray; $\tau_{1}=1.2 \mathrm{~s}$ ( $213 \mathrm{fF}$ ), $\tau_{2}=18.0 \mathrm{~s}$ (1255 fF)]. Right, The same trace in a larger time scale. $\boldsymbol{D}$, Left, Sampled Cm induced by five bursts at $0.4 \mathrm{~Hz}$. Each burst consisted of $50 \mathrm{AP}$-e at $30 \mathrm{~Hz}$. The capacitance decay was fit with a biexponential function [gray; $\tau_{1}=1.1 \mathrm{~s}(93 \mathrm{fF}), \tau_{2}=15.5 \mathrm{~s}(1054 \mathrm{fF})$ ]. Right, The same trace in a larger time scale. $E$, Sampled $\mathrm{Cm}$ induced by $200 \mathrm{AP}-\mathrm{e}$ at $100 \mathrm{~Hz}$ with $10 \mathrm{~mm}$ EGTA in the pipette. The capacitance decay was fit with a monoexponential function (gray; $\tau=24.7 \mathrm{~s}$ ).

\section{Discussion}

The present study described a form of plasticity of endocytosis, an activity-dependent acceleration of endocytosis during repetitive stimulation, including AP-e trains. Acceleration was a result of activation of a rapid form of endocytosis, with $\tau=\sim 1-2 \mathrm{~s}$. It offered a rate of $\sim 129 \mathrm{fF} / \mathrm{s}$ during a pulse train at $1 \mathrm{~Hz}$. It retrieved $\sim 407 \mathrm{fF}$ of membrane, with $\tau=\sim 1.4 \mathrm{~s}$, after a pulse train at 10 $\mathrm{Hz}$ (Fig. $1 B$ ), which offered an initial retrieval rate at $291 \mathrm{fF} / \mathrm{s}$. These high retrieval rates $(129-291 \mathrm{fF} / \mathrm{s})$ corresponded to $\sim 3.3-$ 7.5 vesicles per second at or near an active zone considering that a calyx contains $\sim 600$ active zones (Sätzler et al., 2002) and a vesicle capacitance is $\sim 0.065 \mathrm{fF}$ (Sun et al., 2002). High retrieval rates allow rapid endocytosis to play a dominant role in retrieving vesicles, particularly during intense nerve activity that may empty the functional vesicle pool.

We found that acceleration of endocytosis is mediated by an increase of the calcium influx, in line with previous studies (Klingauf et al., 1998; Sankaranarayanan and Ryan, 2001; Burrone et al., 2002). The fall of the intracellular calcium level after intense stimulation may explain why rapid endocytosis played a minor role after stimulation. These results may explain the paradox that 
slower endocytosis is observed after more intense stimulation (Sankaranarayanan and Ryan, 2000), whereas rapid endocytosis is reported during intense stimulation (Sara et al., 2002; Aravanis et al., 2003) in the hippocampus. Our finding is in contrast to the observation of a constant rate of endocytosis during stimulation, as detected with a $\mathrm{pH}$-sensitive green fluorescence protein, synaptophluourin, in hippocampal synapses (Fernandez-Alfonso and Ryan, 2004). In this study (FernandezAlfonso and Ryan, 2004), however, the time resolution and the signal-to-noise ratio are less than those in the current study. Thus, the apparent difference could be attributable to different sensitivity in detecting rapid endocytosis. Alternatively, synapse specificity may account for the difference. Unlike calyces, calcium shortens the time course of endocytosis at cultured hippocampal synapse without activating a kinetically faster component of endocytosis (Sankaranarayanan and Ryan, 2001).

The linear relation between the time constant of the slow component of endocytosis (Fig. 6) was interpreted as reflecting a rapid endocytic pathway with a limited capacity such that more exocytosis causes saturation of the pathway (Sankaranarayanan and Ryan, 2000). As also noted by Sankaranarayanan and Ryan (2000), the interpretation predicts a linear endocytic time course, in contrast to the experimentally observed exponential time course (Fig. 1). Partly because of this reason, we proposed that membrane accumulation triggers a feedback mechanism to slow down endocytosis (Sun et al., 2002). However, we cannot exclude the possibility that some sort of saturation mechanism, similar but not exactly the one proposed by Sankaranarayanan and Ryan (2000), accounts for the linear relation between the amount of exocytosis and the endocytic time constant.

In summary, we have shown here that after mild stimulation, such as an AP-e, the time course of endocytosis was brief; after an intermediate level of stimulation, such as a $20 \mathrm{~ms}$ depolarization, the time course of endocytosis became slow; and after an intensive stimulation, such as 10 pulses of $20 \mathrm{~ms}$ depolarization at $1-10$ $\mathrm{Hz}$, in addition to a slow time course of endocytosis, a rapid component of endocytosis is activated by calcium. The molecular mechanisms that mediate these different time courses of endocytosis remain not well understood. The slow time course of endocytosis is likely mediated by a clathrin- and dynamin-dependent process (De Camilli et al., 2001). Consistent with this possibility, dialysis of agents that block clathrin and/or dynamin function blocks slow endocytosis in chromaffin cells (Artalejo et al., 1995, 2002), goldfish retinal bipolar nerve terminals (Jockusch et al., 2005), and calyces (Yamashita et al., 2005). Rapid endocytosis has been suggested to be mediated by a clathrin-independent, but dynamin-dependent, process in chromaffin cells (Artalejo et al., 1995, 2002) and goldfish retinal bipolar nerve terminals (Jockusch et al., 2005). In calyces of Held, however, we observed rapid endocytosis in two stimulation conditions, a mild and an intensive stimulation condition. It is unclear whether both are mediated by the same mechanism. It seems more likely that they are mediated by different mechanisms, because rapid endocytosis after intensive but not after mild stimulation was triggered by higher calcium influx. Rapid endocytosis after intensive stimula-

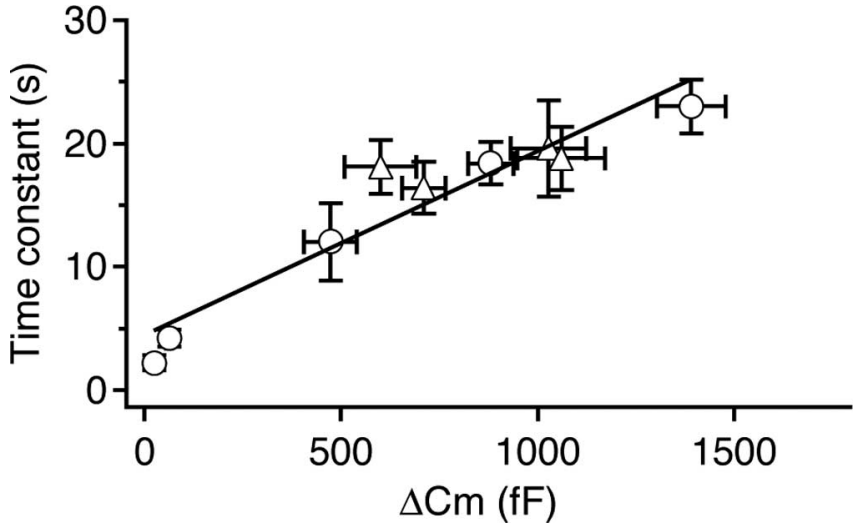

Figure 6. The relationship between the time constant and the amplitude of endocytosis. A plot of endocytosis time constant versus its amplitude after various stimuli is shown. The endocytosis component and stimuli included the slower component of endocytosis after 10 depolarizing pulses at 1 and $10 \mathrm{~Hz}$ (circles), endocytosis after a $20 \mathrm{~ms}$ depolarization (circle), a $1 \mathrm{~ms}$ depolarization to +7 or 15-30 mV (circle), endocytosis after $50 \mathrm{AP}$-e at $100 \mathrm{~Hz}$ (triangle), and the slower component of endocytosis after $200 \mathrm{AP}$-e at $100 \mathrm{~Hz}$ (triangle), $50 \mathrm{AP}$-e at $100 \mathrm{~Hz}$ repeated five times at $1 \mathrm{~Hz}$ (triangle), or $50 \mathrm{AP}$-e at $30 \mathrm{~Hz}$ repeated five times at $0.4 \mathrm{~Hz}$ (triangle). The data were fit with a linear regression line with a slope of $1.4 \mathrm{~s} / 100 \mathrm{fF}$.

tion is perhaps mediated by a clathrin-independent, but dynamin-dependent, process. Rapid endocytosis after mild stimulation is perhaps mediated by the same mechanism as the slow time course of endocytosis, provided that some sort of saturation process discussed above accounts for the slow down of the time course of endocytosis as the stimulation intensity increases. It would be of great interest to distinguish these possibilities in the future.

The main finding of this work is that rapid endocytosis was trigger by an increase of calcium influx during intense nerve activity. Rapid endocytosis played a significant role in retrieving and thus recycling vesicles that may be needed to maintain exocytosis during intense nerve activity. Intense nerve activity may occur in many physiological conditions, such as during sound stimulation, movement, pain perception, and learning (Oertel, 1999; Boraud et al., 2002; Bear, 2003). Rapid endocytosis may thus play a critical role in sustaining synaptic transmission in these physiological conditions. 


\section{References}

Aravanis AM, Pyle JL, Tsien RW (2003) Single synaptic vesicles fusing transiently and successively without loss of identity. Nature 423:643-647.

Artalejo CR, Henley JR, McNiven MA, Palfrey HC (1995) Rapic endocytosis coupled to exocytosis in adrenal chromaffin cells involves $\mathrm{Ca}^{2+}$, GTP, and dynamin but not clathrin. Proc Natl Acad Sci USA 92:8328-8332.

Artalejo CR, Elhamdani A, Palfrey HC (2002) Sustained stimulation shifts the mechanism of endocytosis from dynamin-1-dependent rapid endocytosis to clathrin- and dynamin-2-mediated slow endocytosis in chromaffin cells. Proc Natl Acad Sci USA 99:6358-6363.

Bear MF (2003) Bidirectional synaptic plasticity: from theory to reality. Philos Trans R Soc Lond B Biol Sci 358:649-655.

Boraud T, Bezard E, Bioulac B, Gross CE (2002) From single extracellular unit recording in experimental and human Parkinsonism to the development of a functional concept of the role played by the basal ganglia in motor control. Prog Neurobiol 66:265-283.

Burrone J, Neves G, Gomis A, Cooke A, Lagnado L (2002) Endogenous calcium buffers regulate fast exocytosis in the synaptic terminal of retinal bipolar cells. Neuron 33:101-112.

De Camilli P, Slepnev VI, Shupliakov O, Brodin L (2001) Synaptic vesicle endocytosis. In: Synapses (Cowan WM, Sudhof TC, Stevens CF, eds), pp 217-274. Baltimore: The Johns Hopkins University.

de Lange RP, de Roos AD, Borst JG (2003) Two modes of vesicle recycling in the rat calyx of Held. J Neurosci 23:10164-10173.

Fernandez-Alfonso T, Ryan TA (2004) The kinetics of synaptic vesicle pool depletion at CNS synaptic terminals. Neuron 41:943-953.

Harata N, Pyle JL, Aravanis AM, Mozhayeva M, Kavalali ET, Tsien RW (2001) Limited numbers of recycling vesicles in small CNS nerve terminals: implications for neural signaling and vesicular cycling. Trends Neurosci 24:637-643.

Jockusch WJ, Praefcke GJ, McMahon HT, Lagnado L (2005) Clathrindependent and clathrin-independent retrieval of synaptic vesicles in retinal bipolar cells. Neuron 46:869-878.

Klingauf J, Kavalali ET, Tsien RW (1998) Kinetics and regulation of fast endocytosis at hippocampal synapses. Nature 394:581-585.

Oertel D (1999) The role of timing in the brain stem auditory nuclei of vertebrates. Annu Rev Physiol 61:497-519.

Royle SJ, Lagnado L (2003) Endocytosis at the synaptic terminal. J Physiol (Lond) 553:345-355.

Sankaranarayanan S, Ryan TA (2000) Real-time measurements of vesicle-
SNARE recycling in synapses of the central nervous system. Nat Cell Biol 2:197-204.

Sankaranarayanan S, Ryan TA (2001) Calcium accelerates endocytosis of vSNAREs at hippocampal synapses. Nat Neurosci 4:129-136.

Sara Y, Mozhayeva MG, Liu XR, Kavalali ET (2002) Fast vesicle recycling supports neurotransmission during sustained stimulation at hippocampal synapses. J Neurosci 22:1608-1617.

Sätzler K, Sohl L, Bollmann JH, Borst JGG, Frotscher M, Sakmann B, Lubke JH (2002) Three-dimensional reconstruction of a calyx of Held and its postsynaptic principal neuron in the medial nucleus of the trapezoid body. J Neurosci 22:10567-10579.

Sun JY, Wu LG (2001) Fast kinetics of exocytosis revealed by simultaneous measurements of presynaptic capacitance and postsynaptic currents at a central synapse. Neuron 30:171-182.

Sun JY, Wu XS, Wu LG (2002) Single and multiple vesicle fusion induce different rates of endocytosis at a central synapse. Nature 417:555-559.

Sun JY, Wu XS, Wu W, Jin SX, Dondzillo A, Wu LG (2004) Capacitance measurements at the calyx of Held in the medial nucleus of the trapezoid body. J Neurosci Methods 134:121-131.

Taschenberger H, Leao RM, Rowland KC, Spirou GA, Von Gersdorff H (2002) Optimizing synaptic architecture and efficiency for highfrequency transmission. Neuron 36:1127-1143.

Wong AY, Graham BP, Billups B, Forsythe ID (2003) Distinguishing between presynaptic and postsynaptic mechanisms of short-term depression during action potential trains. J Neurosci 23:4868-4877.

Wu LG (2004) Kinetic regulation of vesicle endocytosis at synapses. Trends Neurosci 27:548-554.

Wu LG, Betz WJ (1996) Nerve activity but not intracellular calcium determines the time course of endocytosis at the frog neuromuscular junction. Neuron 17:769-779.

Wu LG, Borst JGG (1999) The reduced release probability of releasable vesicles during recovery from short-term synaptic depression. Neuron 23:821-832.

Xu T, Binz T, Niemann H, Neher E (1998) Multiple kinetic components of exocytosis distinguished by neurotoxin sensitivity. Nat Neurosci 1:192-200.

Yamashita T, Hige T, Takahashi T (2005) Vesicle endocytosis requires dynamin-dependent GTP hydrolysis at a fast CNS synapse. Science 307: $124-127$. 